\title{
Anti-inflammatory drugs: What is safe?
}

S ome risks of the traditional NSAIDs were well known before the development of selective cyclooxygenase-2 (COX-2) inhibitors (coxibs). Sixteen thousand deaths a year in the United States and 1900 a year in Canada were blamed on NSAID-induced gastric perforations, obstructions and bleeds. NSAID use was complicated also by numerous other side effects, including hypertension, heart failure and renal insufficiency.

The coxibs were developed as a solution to the gastrointestinal (GI) toxicity of NSAIDs. Randomized controlled trials, designed to demonstrate a decreased rate of gastric perforations, obstructions and bleeds, were the first large-scale trials evaluating the safety of coxibs compared with traditional NSAIDs. Unfortunately, the GI advantages of coxibs were offset by an increased rate of cardiovascular events such as myocardial infarction, congestive heart failure and death. These risks led to a reappraisal of the risks of traditional NSAIDs. ${ }^{1,2}$ As a result, one can no longer underestimate the risks of any cyclooxygenase inhibitor, traditional NSAID or coxib. Further, the risks are not limited to long-term use; clinically significant adverse cardiovascular events are noted within the first 30 days of treatment.

Warnings must be duly noted, but what is a doctor to do with a patient who presents with a chronic painful condition? The admonition of doing no harm cannot simply be interpreted as doing nothing. The overall risks have been estimated for populations of patients. However, for the individual patient, estimating his or her risk is the first step in deciding the best therapeutic approach for that patient.

Is an NSAID the best choice? Many regional pain syndromes are short-lived and remit with conservative measures such as relative rest, application of ice and use of acetaminophen. Treatment with an NSAID is not necessary. Acetaminophen, up to $4 \mathrm{~g} / \mathrm{d}$, is as effective as an orally administered NSAID in about $40 \%$ of patients with mild to moderate osteoarthritis.

When an NSAID is required, for example in cases of advanced osteoarthritis or inflammatory arthritis, estimate your patient's baseline risks before starting therapy. If there is an increased risk of gastric toxicity (age $>65$, previous upper GI bleed, concurrent therapy with low-dose ASA, corticosteroids or anticoagulants), consider prescribing a coxib or traditional NSAID with concurrent cytoprotection with misoprostol or a proton pump inhibitor. If your patient has had a recent myocardial infarction or episode of congestive heart failure, do not prescribe an NSAID or coxib. ${ }^{2}$ If your patient has uncontrolled hypertension, do not choose an NSAID or coxib until the blood pressure is well controlled. If the patient has controlled blood pressure that becomes ele- vated while taking an NSAID or coxib, even by $5 \mathrm{~mm}$ Hg systolic, adjust the antihypertensive medications or stop the anti-inflammatory, or both. Check for signs of fluid retention and heart failure. Monitor serum electrolyte and creatinine levels at regular patient visits.

Is there a safe NSAID or coxib? Probably not. Increased risk of cardiovascular events has been noted with all coxibs and, where data are available, with traditional NSAIDs such as diclofenac and ibuprofen. Is naproxen a safe NSAID? The absolute cardiovascular safety of naproxen is uncertain, with the generally positive results of several meta-analyses ${ }^{2}$ tempered by those of several cross-sectional studies and the suspension of ADAPT (the Alzheimer's Disease Antiinflammatory Prevention Trial) because of an elevated cardiovascular risk compared with placebo among patients taking naproxen.

Can the risk be decreased by limiting the daily dose? Doserelated cardiovascular risk has been suggested for both coxibs and traditional NSAIDs. It is reasonable to limit the dose to the lower part of the effective range (e.g., celecoxib $200 \mathrm{mg} / \mathrm{d}$, ibuprofen $1200 \mathrm{mg} / \mathrm{d}$, diclofenac $100 \mathrm{mg} / \mathrm{d}$ ). However, no such relative safety should be assumed in high-risk patients, for whom avoidance of the drugs is the best approach. Does concomitant low-dose ASA therapy protect against cardiovascular events? There are insufficient data to show that it mitigates the cardiovascular risks of coxibs and NSAIDs and adequate data to suggest that it enhances the gastric toxicity of anti-inflammatory drugs.

Patients want effective anti-inflammatory therapy but are worried most about side effects. ${ }^{3}$ To make informed decisions and balance risks and benefits, they need accurate information about the absolute risks, not the relative risks, in clear language, preferably from their doctor and pharmacist rather than from the media. - Jacob Karsh, Professor of Medicine, University of Ottawa, Ottawa, Ont.

This article has been peer reviewed.

Competing interests: Jacob Karsh has received speaker fees from MerckFrosst, Pfizer and Novartis and has participated in research trials for MerckFrosst and Novartis.

\section{REFERENCES}

I. Kearney PM, Baigent C, Godwin J, et al. Do selective cyclo-oxygenase inhibitors and traditional non-steroidal anti-inflammatory drugs increase the risk of atherothrombosis? Meta-analysis of randomized trials. BMJ 2006;332:1302-8.

2. Gislason GH, Jacobsen S, Rasmussen JN, et al. Risk of death or reinfarction associated with the use of selective cyclooxygenase inhibitors and nonselective nonsteroidal anti-inflammatory drugs after acute myocardial infarction. Circulation 2006;II3:2906-I3.

3. Hawker GA, Katz JN, Soloman DH. The patient's perspective on the recall of Vioxx. JRheumatol 2006;33:1082-8. 